\title{
High contrast imaging with an arbitrary aperture: active correction of aperture discontinuities: fundamental limits and practical trade- offs
}

Laurent Pueyo, Colin Norman, Rémi Soummer, Marshall Perrin, Mamadou N'Diaye, et al.

Laurent Pueyo, Colin Norman, Rémi Soummer, Marshall Perrin, Mamadou N'Diaye, Élodie Choquet, Jordan Hoffmann, Alexis Carlotti, Dimitri Mawet, "High contrast imaging with an arbitrary aperture: active correction of aperture discontinuities: fundamental limits and practical trade- offs," Proc. SPIE 9143, Space Telescopes and Instrumentation 2014: Optical, Infrared, and Millimeter Wave, 914321 (28 August 2014); doi: 10.1117/12.2056925

Event: SPIE Astronomical Telescopes + Instrumentation, 2014, Montréal, Quebec, Canada 


\title{
High contrast imaging with an arbitrary aperture: active correction of aperture discontinuities: fundamental limits and practical trade offs
}

\author{
Laurent Pueyo ${ }^{a}$, Colin Norman ${ }^{b}$, Rémi Soummer ${ }^{a}$, Marshall Perrin ${ }^{a}$, Mamadou N'Diaye ${ }^{a}$, \\ Elodie Choquet ${ }^{a}$, Jordan Hoffmann ${ }^{b}$, Alexis Carlotti ${ }^{c}$, Dimitri Mawet ${ }^{d}$ \\ ${ }^{a}$ Space Telescope Science Institute Johns Hopkins University, Baltimore, MD, USA \\ ${ }^{b}$ Department of Physics and Astronomy, Johns Hopkins University, Baltimore, MD, USA \\ ${ }^{c}$ Université Joseph Fourier, Grenoble, France \\ ${ }^{d}$ European Southern Observatory, Vitacura, Chile
}

\begin{abstract}
We present a new method to achieve high-contrast images using segmented and/or on-axis telescopes. Our approach relies on using two sequential Deformable Mirrors to compensate for the large amplitude excursions in the telescope aperture due to secondary support structures and/or segment gaps. We solve the highly non-linear Monge-Ampere equation that is the fundamental equation describing the physics of phase induced amplitude modulation. We determine the optimum configuration for our two sequential Deformable Mirror system and show that high-throughput and high contrast solutions can be achieved using realistic surface deformations that are accessible using existing technologies. We name this process Active Compensation of Aperture Discontinuities (ACAD). We show that for geometries similar to JWST, ACAD can attain at least $10^{-7}$ in contrast and an order of magnitude higher for future Extremely Large Telescopes, even when the pupil features a "missing segment" . Because the converging non-linear mappings resulting from our Deformable Mirror shapes damps near-field diffraction artifacts in the vicinity of the discontinuities this solution is particularly appealing in terms of spectral bandwidth. We present preliminary results that illustrate the performances of ACAD in the presence of diffraction for apertures for with secondary support structures of varying width and argue that the ultimate contrast achieved can by combining ACAD with modern wavefront control algorithms.
\end{abstract}

Keywords: planetary systems - techniques: coronagraphy, wavefront control

\section{INTRODUCTION}

The coronagraphs that will equip upcoming Ex-AO instruments on 8 meter class telescopes have been designed for contrasts of at most $\sim 10^{-7}$. Secondary support structures (or spiders: 4 struts each $1 \mathrm{~cm}$ wide, $\sim 0.3 \%$ of the total pupil diameter in the case of Gemini South) have a small impact on starlight extinction at such levels of contrasts. In this case, coronagraphs have thus been optimized on circularly symmetric apertures, which only take into account the central obscuration (1). However, high-contrast instrumentation on future observatories will not benefit from such gentle circumstances. ELTs will have to support a substantially heavier secondary than 8 meter class observatories do, and over larger lengths: as a consequence the relative area covered by the secondary support will increase by a factor of 10 (30 cm wide spiders, occupying $\sim 3 \%$ of the pupil diameter in the case of TMT). This will degrade the contrast of coronagraphs only designed for circularly obscured geometries by a factor $\sim 100$, when the actual envisioned contrast for an ELT exo-planet imager can be as low as $\sim 10^{-8}(2)$. While the trade-offs associated with minimization of spider width in the space-based case have yet to be explored, secondary support structures will certainly hamper the contrast depth of coronagraphic instruments of such observatories at levels that are well above the $10^{10}$ contrast requirement. Coronagraphs for off-axis telescopes take advantage of the pupil symmetry to reach a theoretical contrast of ten orders of magnitude $(3 ; 4 ; 5 ; 6 ; 7 ; 8 ; 9)$. However, using obscured on-axis and/or segmented apertures take full advantage of the limited real estate associated with

Further author information: (Send correspondence to Laurent Pueyo)

Laurent Pueyo: E-mail: pueyo@stsci.edu

Space Telescopes and Instrumentation 2014: Optical, Infrared, and Millimeter Wave, edited by Jacobus M. Oschmann, Jr., Mark Clampin, Giovanni G. Fazio, Howard A. MacEwen, Proc. of SPIE Vol. 9143, 914321 - ( 2014 SPIE

CCC code: $0277-786 \mathrm{X} / 14 / \$ 18 \cdot$ doi: $10.1117 / 12.2056925$

Proc. of SPIE Vol. $9143914321-1$ 
a given launch vehicle and can allow larger apertures that increase the scientific return of space-based direct imaging survey. Devising a practical solution for broadband coronagraphy on asymmetric, unfriendly apertures is thus an outstanding problem in high contrast instrumentation. The purpose of the present paper is to introduce a family of practical solutions to this problem and features its performances on a few characteristic examples.

Our method takes advantage of state-of-the art Deformable Mirrors in modern high-contrast instruments to address the problem of pupil amplitude discontinuities for on-axis and/or segmented telescopes. Indeed, coronagraphs are not sufficient to reach the high contrast required to image faint exo-planets: wavefront control is needed to remove the light scattered by small imperfections on the optical surfaces (10). Over the past eight years, significant progress has been made in this area, both in the development of new algorithms $(11 ; 12)$ and in the experimental demonstration of high-contrast imaging with a variety of coronagraphs $(12 ; 13 ; 14 ; 15)$. These experiments rely on a system with a single Deformable Mirror which is controlled based on diagnostics downstream of the coronagraph, either at the science camera or as close as possible to the end detector $(16 ; 17)$. Such configurations are well suited to correct phase wavefront errors arising from surface roughness but have limitations in the presence of pure amplitude errors (reflectivity), or phase-induced amplitude errors, which result from the propagation of surface errors in optics that are not conjugate to the telescope pupil $(18 ; 19)$. Indeed a single DM can only mimic half of the spatial frequency content of amplitude errors and compensate for them only on one half of the image plane (thus limiting the scientific field of view) over a moderate bandwidth. In theory, architectures with two sequential Deformable Mirrors, can circumvent this problem and create a symmetric broadband high contrast PSF $(18 ; 19)$. The first demonstration of symmetric dark hole was reported in (20) and has since been generalized to broadband by (21): a wavefront control system composed of two sequential Deformable Mirrors is currently the baseline architecture of currently envisioned coronagraphic spacebased instruments $(22 ; 23)$ and ELT planet imagers (2). The purpose of our study is to demonstrate that two Deformable Mirror (DM for the remainder of this paper) can also mitigate the impact of the pupil asymmetries, such as spiders and segments, on contrast and thus enable high contrast on unfriendly apertures.

\section{PHYSICS OF AMPLITUDE MODULATION}

\subsection{Ray optics}

In this section we briefly review the results in (24). We assume that one can design azimuthally symmetric coronagraphs which mitigate the impact of the secondary obscuration itself $(24 ; 25 ; 26)$. We demonstrate that well controlled DMs can circumvent the obstacle of spiders and segment gaps. We first set-up our notations and review the physics of phase to amplitude modulation. We consider the system represented on Fig. 1 where two sequential DMs are located between the telescope aperture and the entrance pupil of the coronagraph. In this configuration, the telescope aperture and the pupil apodizer are not in conjugate planes. Without loss of generality we work under the "folded" assumption, where the DMs are not tilted with respect to the optical axis and can be considered as lenses of index of refraction -1 (as discussed in (27). In the scalar approximation the relationship between the incoming field, $E_{D M 1}(x, y)$, and the outgoing field, $E_{D M 2}\left(x_{2}, y_{2}\right)$, is given by the diffractive Huygens Integral:

$$
\begin{aligned}
& E_{D M 2}\left(x_{2}, y_{2}\right)=\frac{1}{i \lambda Z} \int_{\mathcal{A}} E_{D M 1}(x, y) e^{i \frac{2 \pi}{\lambda} Q\left(x, y, x_{2}, y_{2}\right)} d x d y \\
& Q\left(x, y, x_{2}, y_{2}\right)=h_{1}(x, y)-h_{2}\left(x_{2}, y_{2}\right)+\sqrt{\left(x-x_{2}\right)^{2}+\left(y-y_{2}\right)^{2}+\left(Z+h_{1}(x, y)-h_{2}\left(x_{2}, y_{2}\right)\right)^{2}}
\end{aligned}
$$

where $\mathcal{A}$ corresponds to the telescope aperture, $Z$ is the distance between between the two DMs, $h_{1}$ and $h_{2}$ are the shapes of DM1 and DM2 respectively, $\lambda$ is the wavelength and $S\left(x, y, x_{2}, y_{2}\right)$ is the free space propagation between the DMs. We recognize that two sequential DMs act as a pupil remapping unit similar to PIAA coronagraph (28) whose ray optics equations were first derived by (29). We briefly state the notation used to describe such an optical system as introduced in (30). The functions $\left(f_{1}, g_{1}\right)$ and $\left(f_{2}, g_{2}\right)$ trace the geometrical remapping between a point $\left(x_{1}, y_{1}\right)$ at the surface of DM1 and a point $\left(x_{2}, y_{2}\right)$ at the surface of DM2 and are 


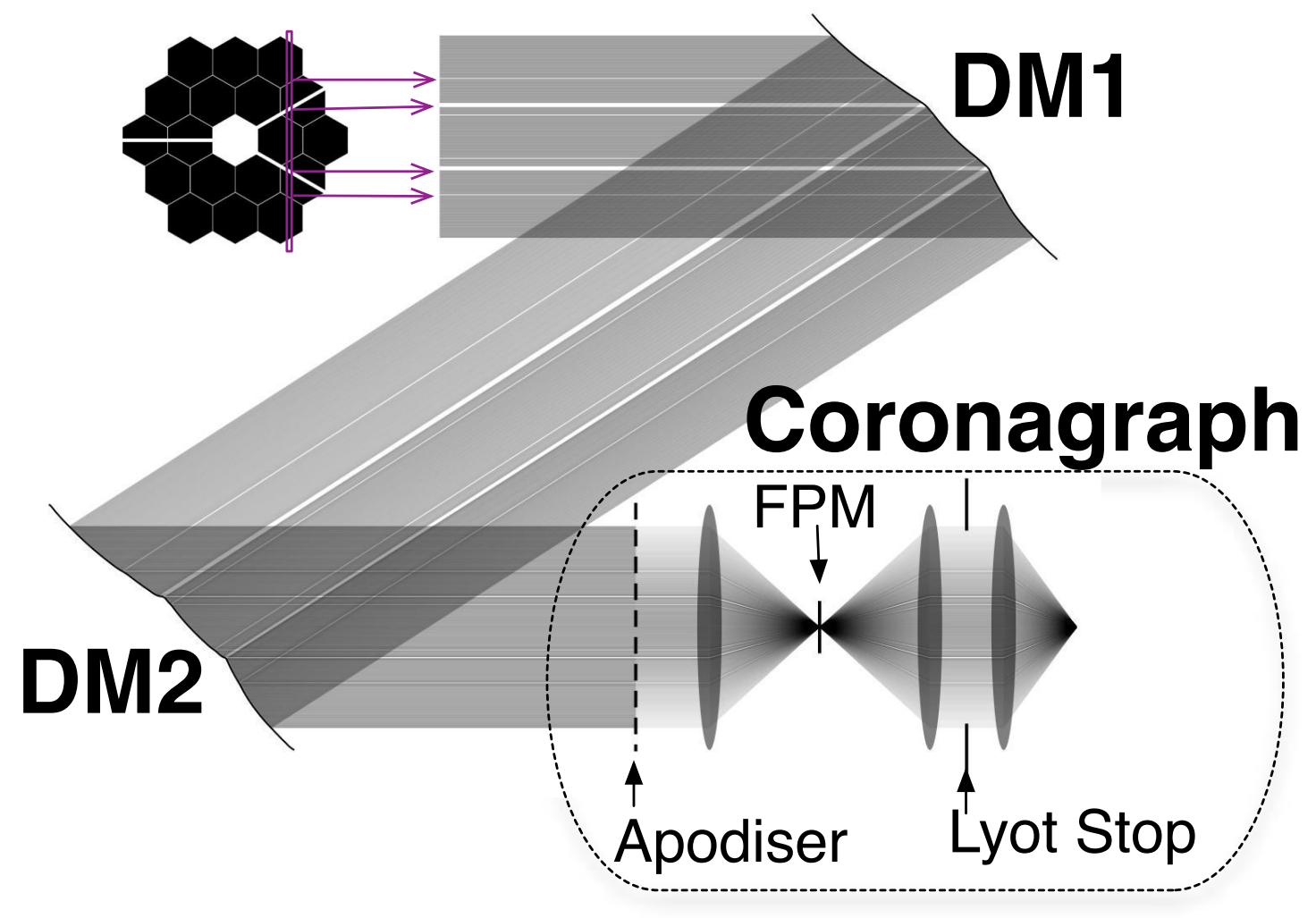

Figure 1. Schematic of the optical system considered: the telescope apertures is followed by two sequential Defromable Mirrors (DMs) in non-conjugate planes whose purpose is to remap the pupil discontinuities. The beam then enters a coronagraph to suppress the bulk of the starlight: in this figure we show an Apodized Lyot Pupil Coronagraph (APLC). This is the coronagraphic architecture we consider for the remainder of the paper but we stress that the method presented herein is applicable to any coronagraph.

tied to the DM surfaces by the following equations:

$$
\begin{aligned}
& x_{i}\left(x_{j}, y_{j}\right)=f_{i}\left(x_{j}, y_{2}\right),\left.\quad \frac{\partial h_{j}}{\partial x}\right|_{\left(x_{j}, y_{j}\right)}=\frac{x_{j}-f_{i}\left(x_{j}, y_{j}\right)}{Z}, \quad(i, j) \in\{(1,2),(2,1)\} \\
& y_{i}\left(x_{j}, y_{j}\right)=g_{i}\left(x_{j}, y_{j}\right),\left.\quad \frac{\partial h_{j}}{\partial y}\right|_{\left(x_{j}, y_{j}\right)}=\frac{y_{j}-g_{i}\left(x_{j}, y_{j}\right)}{Z}, \quad(i, j) \in\{(1,2),(2,1)\}
\end{aligned}
$$

\subsection{Diffractive optics}

In (30) we showed that one could approximate the propagation integral in Eq. 1 by taking in a second order Taylor expansion of $Q\left(x, y, x_{2}, y_{2}\right)$ around the rays that trace $\left(f_{1}\left(x_{2}, y_{2}\right), g_{1}\left(x_{2}, y_{2}\right)\right)$ to $\left(x_{2}, y_{2}\right)$. In this case the relationship between the fields at DM1 and DM2 is:

$$
E_{D M 2}\left(x_{2}, y_{2}\right)=\left.\frac{e^{\frac{2 i \pi}{\lambda} Z}}{i \lambda Z}\left\{\int_{\mathcal{A}} E_{D M 1}(x, y) e^{\frac{i \pi}{\lambda Z}\left[\frac{\partial f_{2}}{\partial x}\left(x-x_{1}\right)^{2}+2 \frac{\partial g_{2}}{\partial x}\left(x-x_{1}\right)\left(y-y_{1}\right)+\frac{\partial g_{2}}{\partial y}\left(y-y_{1}\right)^{2}\right]} d x d y\right\}\right|_{\left(x_{1}, y_{1}\right)}
$$

In (24) we showed that this could be reduced to the what we called the Streched-Remapped Fresnel approximation:

$$
E_{D M 2}\left(x_{2}, y_{2}\right)=\left.\left\{\sqrt{|\operatorname{det}[J]|} \int_{\mathcal{F P}} \widehat{E_{D M 1}}(\xi, \eta) e^{i 2 \pi\left(\xi f_{1}+\eta g_{1}\right)} e^{-i \frac{\pi \lambda Z}{\operatorname{det}[J]}\left(\frac{\partial g_{1}}{\partial y} \xi^{2}+\frac{\partial f_{1}}{\partial x} \eta^{2}\right)} d \xi d \eta\right\}\right|_{\left(x_{2}, y_{2}\right)}
$$


This approximate expression of the diffracted field is very similar to the Fresnel propagation, derived from Eq. 1 assuming that the the large quantity is the distance $\mathrm{Z}$ between the two DMs:

$$
E_{D M 2}\left(x_{2}, y_{2}\right)=\frac{1}{i \lambda Z} e^{i \frac{4 \pi}{\lambda} h_{2}\left(x_{2}, y_{2}\right)} \int_{\mathcal{A}} E_{D M 1}(x, y) e^{i \frac{4 \pi}{\lambda} h_{1}(x, y)} e^{i \frac{2 \pi}{\lambda Z}\left[(x-x 2)^{2}+(y-y 2)^{2}\right]} d x d y
$$

When the mirror's deformations are very small compared to both the wavelength and $D^{2} / Z$, the net effect of the wavefront disturbance created by DM1 is an amplitude ripple at DM2. This effect can be derived using either using Eq. 5 of Eq. 6. Indeed if $h_{1}(x, y)=\lambda \epsilon \cos \left(\frac{2 \pi}{D}(m x+n y)\right), h_{2}(x, y)=-h_{1}(x, y)$, with $\epsilon \ll 1$, then the outgoing field is to first order:

$$
E_{D M 2}\left(x_{2}, y_{2}\right) \propto \frac{\pi \lambda Z\left(m^{2}+n^{2}\right)}{D^{2}} \lambda \epsilon \cos \left(\frac{2 \pi}{D}\left(m x_{2}+n y_{2}\right)\right) .
$$

This phase-to-amplitude coupling is a well known optical phenomenon called Talbot imaging and was introduced to the context of high contrast imaging by (18). In the small deformation regime, the phase on DM1 becomes an amplitude at DM2 according to the coupling in Eq. 7. When two sequential DMs are controlled to cancel small amplitude errors, as in (20), they operate in this regime. Note, however, that the coupling factor scales with wavelength (the resulting amplitude modulation is wavelength independent, but the coupling scales as $\lambda$ ): this formalism is thus not applicable to our case, for which we are seeking to correct large amplitude errors (secondary support structures and segments) with the DMs. When using Eq. 7 in the wavefront control scheme outlined in (20) to correct aperture discontinuities, this weak coupling results in large mirror shapes that lie beyond the range of the linear assumption made by the DM control algorithm: because phase to amplitude conversion is fundamentally a very non-linear phenomena, these descending gradient methods $(11 ; 12 ; 20)$ are not readily suitable to find DM shapes that mitigate apertures discontinuities.

In order to account for non linearities associated with the problem of compensation of aperture discontinuities we follow a two step approach:

- We first calculate the best possible remapping solution in the ray optics regime. This is an "open loop" solution as our solver does not use any post-coronagraph image plane based metric.

- We use diffractive models in order to propagate the actual telescope wavefront in the spectral bandwidth of interest through both the two DMs ACAD unit and the coronagraph.

- We finally apply the aforementioned quasi-linear wavefront control methods in order to adjust the DM shape and reach deeper contrasts.

In Section 3 we first present results in the ray optics regime. In Section 4 we discuss our choice of diffractive model and present preliminary results that illustrate the potential of the method outlined above.

\section{EXAMPLES IN THE RAY OPTICS REGIME}

Our first step consists of calculating the ray optics remapping, under the constraint that the DM surface can be expanded in a finite number of Fourier modes. We mitigate the fact that this representation is not fully realistic by using the influence function representations in the algorithms discussed in Section 4 . We refer the reader to Pueyo and Norman(24) for details on the non-linear solver for the DM shapes and here only discuss our results.

\subsection{JWST like geometry}

\subsubsection{Remapped aperture}

Since the curvature of the DMs is limited by the number of modes $N$, our solution does not fully map out the discontinuities induced by the secondary supports and the segments. However, they are significantly thinner and one can expect that their impact on contrast will be attenuated by orders of magnitude. In order to quantify the final coronagraphic contrasts of our solution we then propagate it through an APLC coronagraph optimized to accommodate for a circular central obscuration. The results on this section assume that ray optics holds perfectly and that there are no diffractive artifacts associated with $A C A D$, in the next section we will discuss the impact of diffraction on bandwidth. 

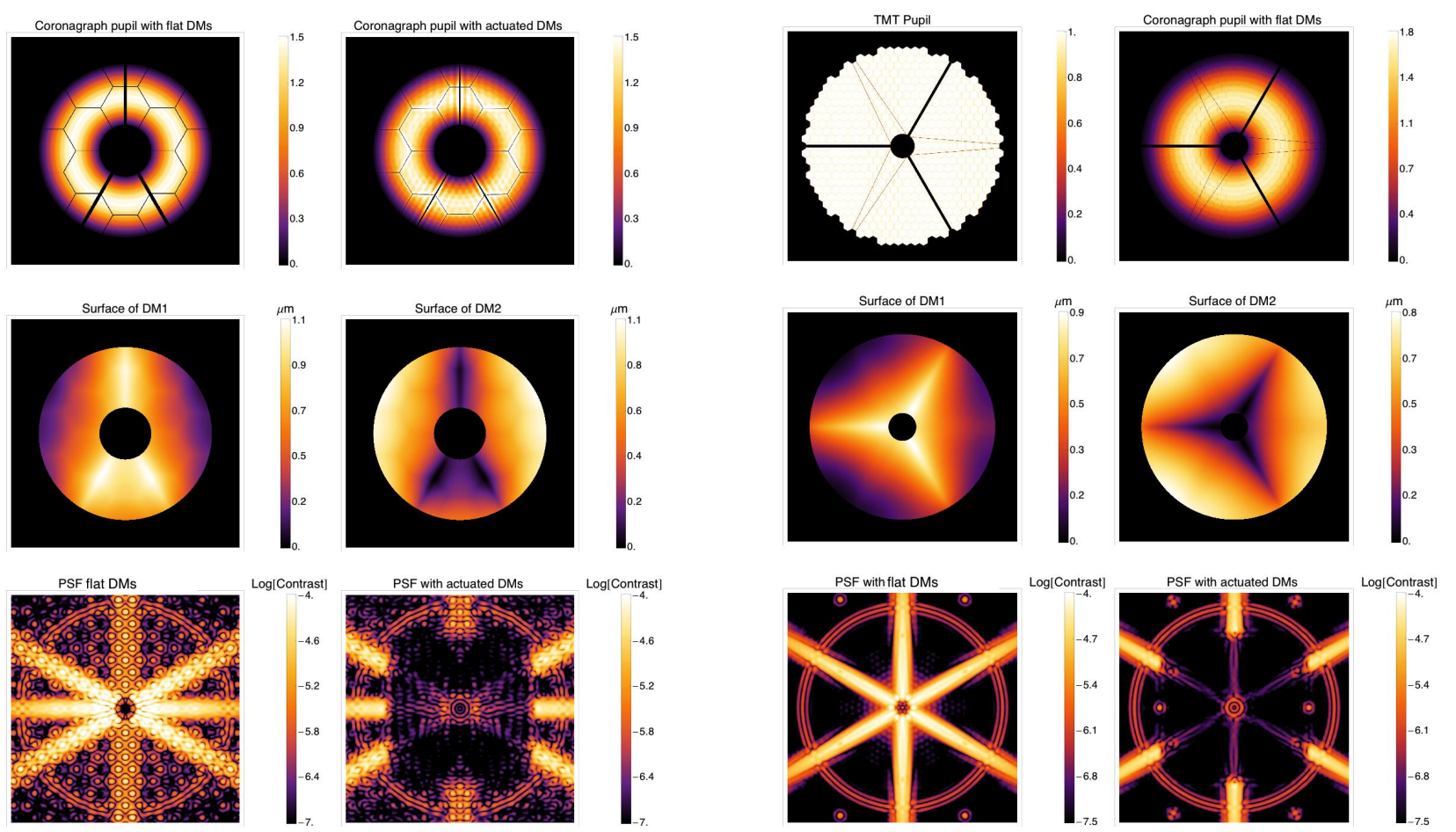

Figure 2. Left: Results obtained when applying our approach to a geometry similar to JWST . We used two 3 cm DMs of 64 actuators separated by $1 \mathrm{~m}$. Their maximal surface deformation is $1.1 \mu \mathrm{m}$, well within the stroke limit of current DM technologies. The residual light in the corrected PSF follows the secondary support structures and can potentially be further cancelled by controlling the DMs using an image plane based cost function. Right: Results obtained when applying our approach to a TMT geometry. We used two $3 \mathrm{~cm}$ DMs of 64 actuators separated by $1 \mathrm{~m}$. Their maximal surface deformation is $0.9 \mu \mathrm{m}$, well within the stroke limit of current DM technologies. The final contrast is below $10^{7}$, in a regime favorable for direct imaging of exo-planets with ELTs.

\subsubsection{Contrast}

We have illustrated each step of the calculation of the DM shapes using a geometry similar of JWST. This configuration is somewhat a conservative illustration of an on-axis segmented telescope as it features thick secondary supports and a "small" number of segments whose gaps diffract light in regions of the image plane close to the optical axis (the first diffraction order of a six hexagons structure is located at $\sim 3 \lambda / D$ ). In order to assess the performances of ACAD on such an observatory architecture we chose to use a coronagraph designed around a slightly oversized secondary obscuration of diameter $0.25 D$, with a focal plane mask of diameter $8 \lambda / D$, an IWA of $5 \lambda / D$ and an OWA of $30 \lambda / D$. The field at the entrance of the coronagraph after remapping by the DMs is shown on the top right panel of Fig. 2. The DM surfaces, calculated assuming 64 actuators across the pupil ( $N=64$ in the Fourier expansion) and DMs of diameter $3 \mathrm{~cm}$ separated by $Z=1 \mathrm{~m}$, are shown on the middle panel of Fig. 2. They are well within the stroke limit of current DM technologies. On Fig. 3 we then show how to further the contrast of this solution, even in the presence of wavefront errors, by superposing a quasi-linear wavefront control algorithm to this solution. These results illustrate that even with a very unfriendly aperture similar to JWST one can obtain contrasts as high as envisioned for upcoming Ex-AO instruments, which have been designed for much friendlier apertures. While we certainly do not advocate to use such a technique on JWST, this demonstrates that ACAD is a powerful tool for coronagraphy with on-axis segmented apertures.

\subsection{TMT Geometry}

We now discuss the case of Extremely Large Telescopes and provide an illustration using the example of the Thirty Meter Telescope. The pupil consists of 37 segments across in the longest direction and a secondary of diameter $\sim 0.12 D$ which is held by three main thick struts and six thin cables. As seen in the right panel of 
Fig. 2 the impact of segment gaps is minor as they diffract light beyond the OWA of the coronagraph. When using a coronagraph with a larger OWA the segment gaps will have to be taken into account, and will have to be mitigated using DMs with a larger number of actuators. In order to obtain first order estimates of the performances of ACAD on the aperture geometry shown on the top right panel of Fig. 2, we chose to use a coronagraph designed around a slightly oversized secondary obscuration of diameter $0.15 \mathrm{D}$, with a focal plane mask of $6 \lambda / D$ diameter, an IWA of $4 \lambda / D$ and an OWA of $30 \lambda / D$. The DM surfaces, calculated assuming 64 actuators across the pupil ( $N=64$ in the Fourier expansion) and DMs of diameter $3 \mathrm{~cm}$ separated by $Z=1 \mathrm{~m}$. They are well within the stroke limit of current DM technologies. The final PSF is shown on the bottom right panel of Fig. 2 and features a high contrast dark hole with residual diffracted light at the location of the spiders' diffraction structures. The impact on coronagraphic contrast of secondary supports was thoroughly studied by (31). They concluded that under a $90 \%$ Strehl ratio, the contrast in most types of coronagraphs is driven by the secondary support structures to levels ranging from $10^{-4}$ to $10^{-5}$. This, in turn, leads to a final contrast after post-processing (called Differential Imaging) of $\sim 10^{-7}-10^{-8}$. Fig. 4 shows that using ACAD on an ELT pupil yields contrasts before any post-processing which are comparable to the ones obtained by (31) after Differential Imaging. On Fig 5 we also how the DMs can be controlled to actively mitigate for the absence of segments in an ELT pupil and retrieve contrast similar to the case of a full pupil. Being able to operate at deep contrast even without a full ELT pupil has profound implications on the operations of such futures observatories: this enables scientific operations to occur while some segments go through their regular maintenance cycle and opens up the possibility to observe with a temporarily uncontrollable segment (provided that it can be titled away from the main optical path). This shows that not only ACAD provides solutions for deep contrast in future observatories but also opens a wide range of operational possibilities via its adaptability to arbitrary pupil geometries.

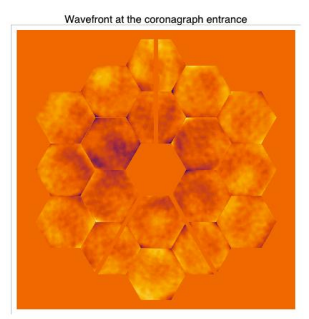

Detormation of DM2 $2 \mathrm{~A}_{2}$

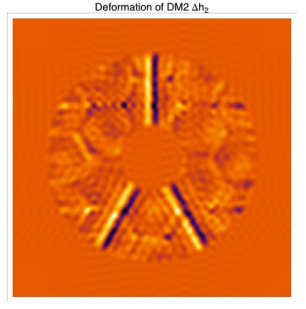

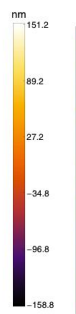

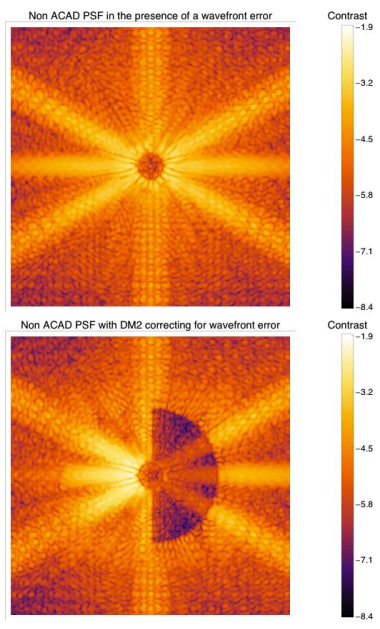

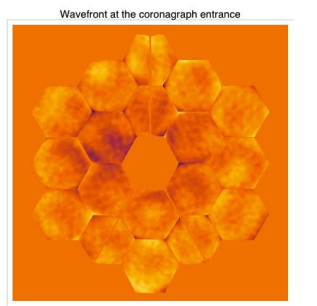

Delomation of DM2 shes

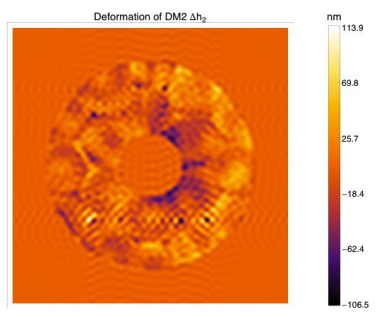

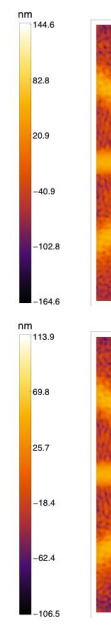

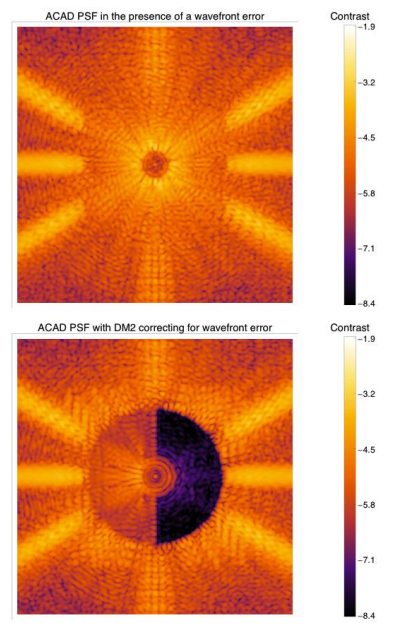

Figure 3. Broadband wavefront correction (20\% bandwidth around $700 \mathrm{~nm}$ ) with a single DM in segmented telescope with discontinuous surface errors. Top: Top Left: wavefront before the coronagraph. Top Right: broadband aberrated PSF with DM at rest. Bottom Left: DM surface resulting from the wavefront control algorithm. Bottom Right: broadband corrected PSF. Note that the wavefront control algorithm seeks to compensate for the diffractive artifacts associated with the secondary support structures: it attenuates them on the right side of the PSF while it strengthens them on the left side of the PSF. As a result the DM surface becomes too large at the pupil spider's location and the quasi-linear wavefront control algorithm eventually diverges. Bottom: he surface of the first DM is set according to the ACAD equations. The surface of the second DM is the sum of the ACAD solution and a small perturbation calculated using a quasilinear wavefront control algorithm . Top Left: wavefront before the coronagraph. Note that the ACAD remapping has compressed the wavefront errors near the struts and the segment gaps. Top Right: broadband aberrated PSF with DMs set to the ACAD solution. Bottom Left: perturbation of DM2's surface resulting from the wavefront control algorithm. Bottom Right: broadband corrected PSF. The wavefront control algorithm now yields a DM surface that does not feature prominent deformations at the location of the spiders. Most of the DM stroke is located at the edge of the segments, at location of the wavefront discontinuities. There, the DM surface eventually becomes too large and the quasi-linear wavefront control algorithm diverges. However this occurs higher contrasts than in the absence of ACAD. 

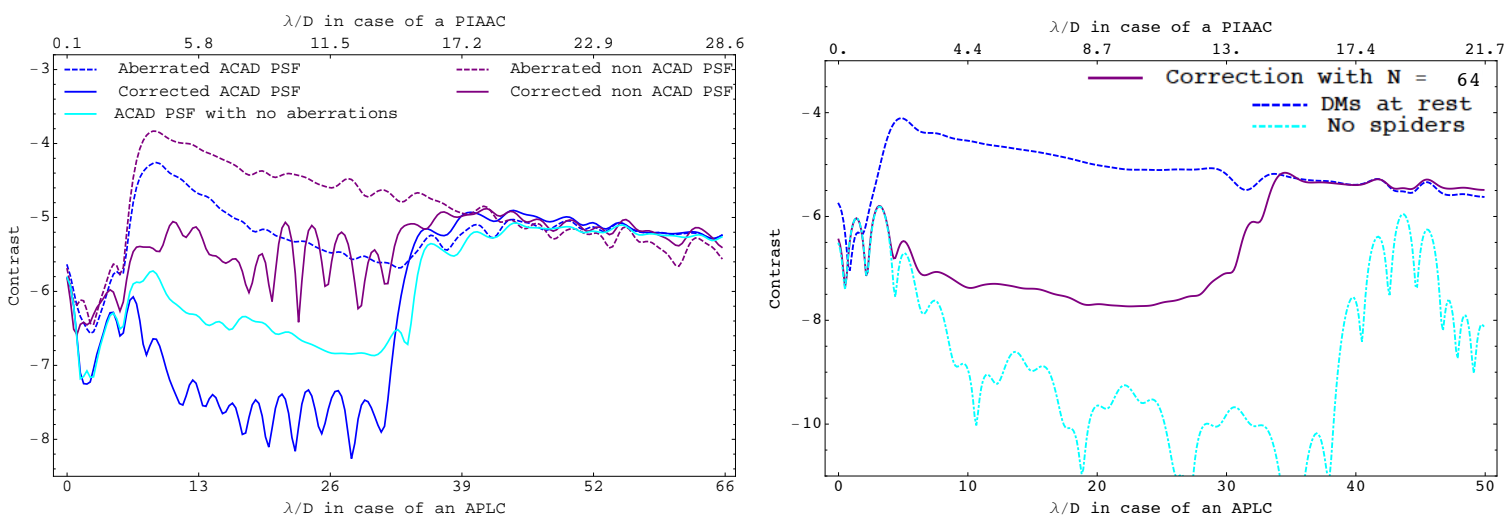

Figure 4. Left: radial average obtained when applying ACAD. We used two $3 \mathrm{~cm}$ DMs of 64 actuators separated by $1 \mathrm{~m}$. Their maximal surface deformation is $0.9 \mu \mathrm{m}$, well within the stroke limit of current DM technologies. The final contrast is below $10^{7}$, in a regime favorable for direct imaging of exo-planets with ELTs. Since ACAD removes the bulk of the light diffracted by the asymmetric aperture discontinuities, the final contrast can be enhanced by controlling the DMs using and image plane based metric. Right: Radial average in the half dark plane of the PSFs on Fig 3. In the presence of wavefront discontinuities corrected using a continuous membrane DM, ACAD still yields, over a $20 \%$ bandwidth around $700 \mathrm{~nm}$, PSF with a contrast 100 larger than in a classical segmented telescope. Moreover this figure illustrates that since it is based on a true image plane metric, the wavefront control algorithm can be used ( within the limits of its linear regime) to improve upon the ACAD DM shapes derived solving the Monge Ampere Equation.
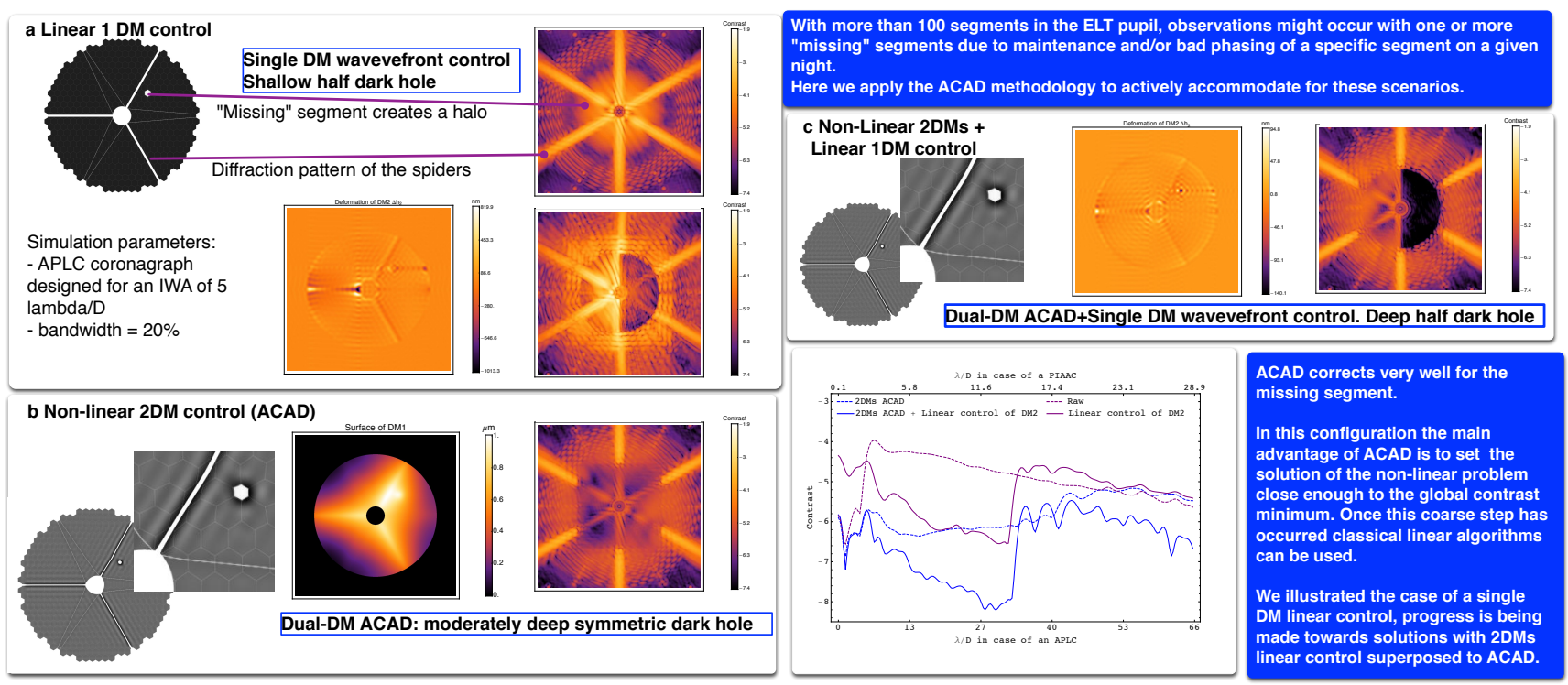

Figure 5. Case of a missing ELT segment: ACAD corrects very well for the missing segment. In this configuration the main advantage of ACAD is to set the solution of the non-linear problem close enough to the global contrast minimum. Once this coarse step has occurred classical linear algorithms can be used. We illustrated the case of a single DM linear control.

\section{CASE STUDY IN THE DIFFRACTIVE REGIME}

\subsection{Deformable Mirrors placement vs stroke}

As discussed in Pueyo and Norman (24), a given geometry we first solve the normalized Monge Ampere Equation over a finite set of DM modes (fourier basis set of influence functions). This solver includes a constraint in maximum curvature and maximum slope for the DMs. We consider two possible "six -spiders" geometries that 

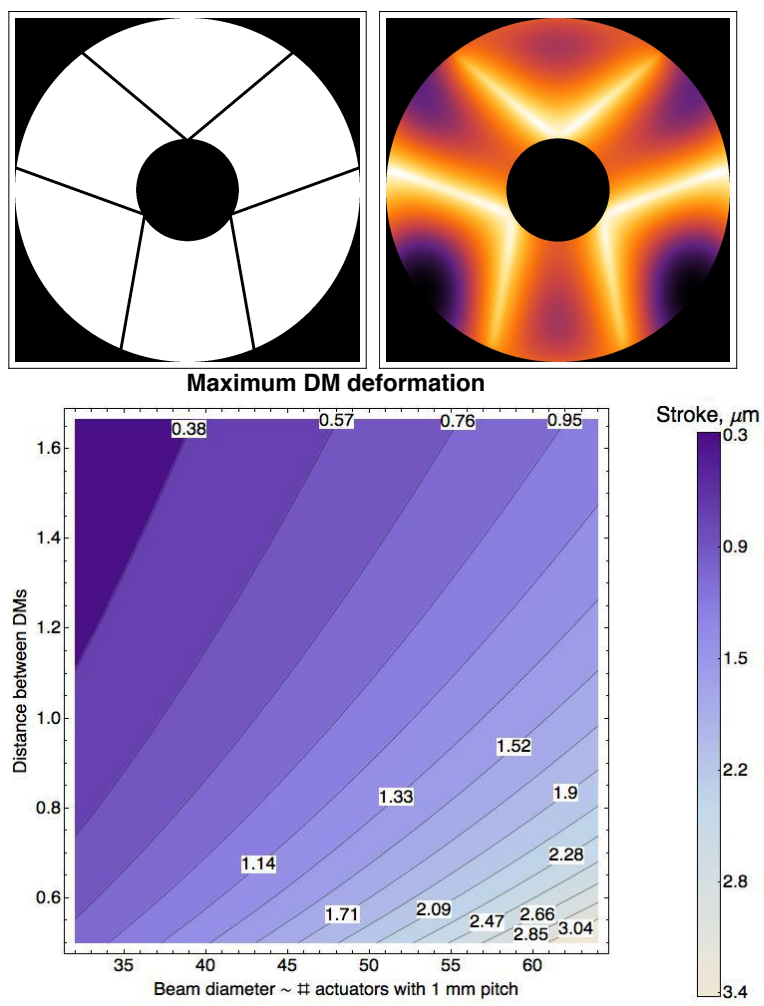
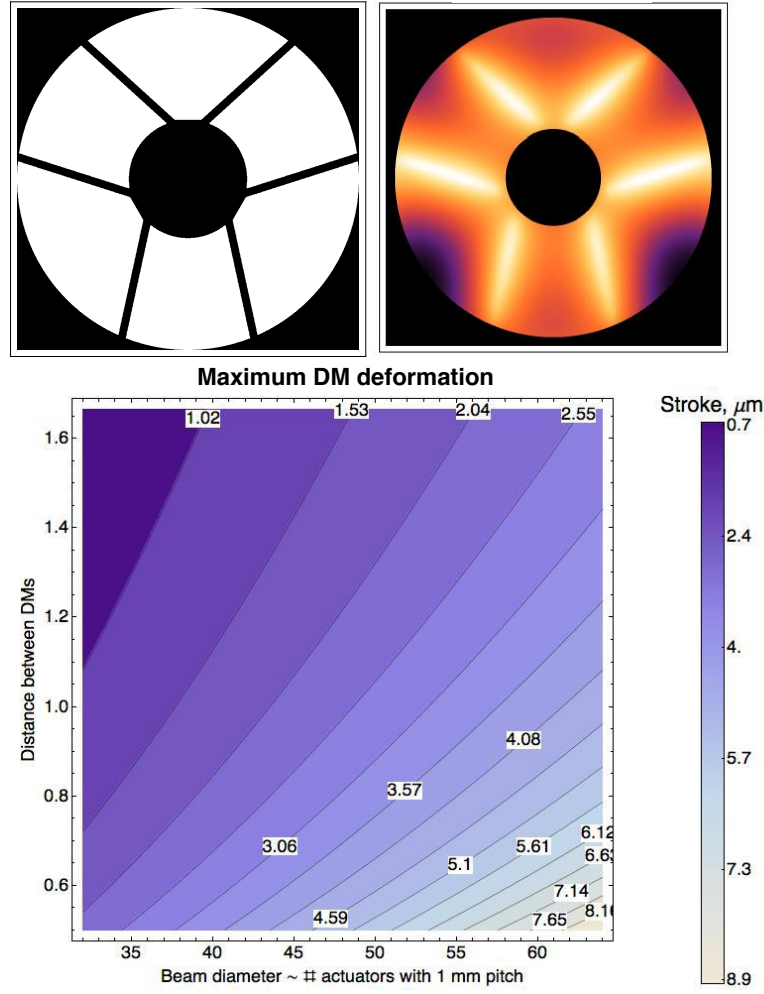

Figure 6. Left: Case of Thin struts. There exist a wide range of DMs geometries for which the maximal ACAD stroke is below the $2 \mu \mathrm{m}$ limit. This leaves ample room in the DM stroke budget for other tasks such as wavefront control. Right: Case of Thick struts. Here the range of DMs geometries for which the maximal ACAD stroke is below the $2 \mu \mathrm{m}$ limit is reduced but favorable geometries can be found

we will call "thin struts" and "thick struts". These have respective strut widths of $2.4 \mathrm{~cm}$ and $5 \mathrm{~cm}$, assuming a 2.4 meter pupil. We consider DM properties consistent with currently available piezo-electric DMs, with actuators separated by $1 \mathrm{~mm}$, and the number of actuators across the pupil ranging from 32 to 64 (e.g $D=32$ $\mathrm{mm}$ to $64 \mathrm{~mm}$ ) and DM separations ranging from $0.5 \mathrm{~m}$ to $1.6 \mathrm{~m}$. Our results are shown on Fig. 6 . As one would expect intuitively DM stroke increases with the thickness of the secondary support structures: in normalized coordinates we find that the max stroke required by ACAD is 2.7 larger when going from thin $(2.4 \mathrm{~cm})$ to thick $(5 \mathrm{~cm})$ struts. Next we study the performances of the method outlined in Section 2 when applied to a continuum of support structures widths in this "six -spiders" configuration. To do so we used Fig. 6 to chose a geometry capable of achieving DM strokes achievable using current piezo-electric technologies: two DMs of $3.2 \mathrm{~cm}(32$ actuators across) separated by one meter. In the future we will further explore this part of the parameter space associated with the ACAD optical layout.

\subsection{Model for diffraction}

Diffractive studies of the Phase Induced Amplitude Apodization coronagraph $(32 ; 33 ; 34)$ have shown that in the case of strong pupil remapping the Fresnel approximation was not adequate to capture the diffracts physics between the two aspheric surfaces, even if it is sufficient in the case of amplitude wavefront control (20). ACAD operates in an intermediate regime for which the remapping formalism ought to be considered, but yields DM shapes that deviate mush less from flat surfaces that PIAA mirrors. In Pueyo and Norman (24) we discussed the diffractive properties of ACAD in the SR-Fresnel regime, and provided on qualitative arguments regarding its applicability to the problem at hand. We concluded that while further numerical was needed, the fact that ACAD main feature was to compress the beam near the location of the secondary support structures implied a minimal impact on the chromaticity (illustrated on Fig. 7). We recently revisited this problem and confirmed 
our intuition regarding the minimal impact of beam compression on chromaticity via an exhaustive battery of numerical tests. However we were able that our initial intuition regarding the modeling method was misguided and we unambiguously established that in the case of ACAD DM Deformation, Fresnel models actually provide a higher fidelity description of the diffracted field than SR-Fresnel. Indeed, SR-Fresnel captures accurately the diffracted amplitude at DM2 (second order terms in OPD) it ignores third order terms in OPD that yield a small, but non negligible contribution to the phase at DM2. Fresnel on the other hand fully captures these third order contributions, and while it only provides an approximate description of the second order (amplitude) OPD terms, this approximation yield sufficient level of accuracy (driven by $\sim 10^{10}$ contrast requirements, for ACAD like DM shapes (this statement is not true for PIAA like shapes). We will present the details of these findings in a future communication. Overall using Fresnel based models does not change the conclusions Fig. 7. Moreover, because of the numerical tractability, such models greatly facilitate the simulations in this section and the use of more advanced wavefront control algorithms once the ray optics open loop DM shapes have been determined.

\subsection{Description of Numerical experiment}

Here we discuss the outcome of a numerical study aimed at quantifying the impact of the width of secondary support structures on the broadband contrast yielded by ACAD solutions. To do so we first compute ray optics open loop DM shapes for a series of "six spiders" telescope pupil of varying width. For each pupil configuration we compute a suite of open loop ACAD mirror shapes for which we vary the "agressivemess" of the remapping. We then choose to use a coronaraph that is an Apodized Pupil Lyot Coroangraph with the following performances: contrast $10^{-9}$ over $10 \%$ bandwidth around $600 \mathrm{~nm}$, OWA $=32 \lambda / D$, IWA $=5 \lambda / D, \mathrm{~T} \sim 40 \%$ when the apodization is implemented using a classical apodizer, OWA $\sim 16 \lambda / D$, IWA $\sim 2.5 \lambda / D, \mathrm{~T} \sim 100 \%$ when the apodization is implemented using a pupil remapping apodizer. This coronagraph was designed according to a novel method developed by our team, also described in the present proceedings. The secondary obstruction for this tests case cover $36 \%$ of the telescope aperture diameter in the input pupil and $50 \%$ of the telescope aperture diameter in the Lyot plane. Note that this choice of coronagraph is quite conservative: in the future we will conduct a similar study using the more aggressive designs such as the ones in Mawet at al. [MAWET] and Fogarty et al. [FOGARTY]. We combine each pupil configuration and each ACAD open loop shapes within the suite of remapping functions (from "not aggressive" to "very aggressive") in order to calculate the broadband contrast of each configuration. To do so we use a Fresnel model of the ACAD unit, a Fraunhofer model of the cornagraph with the relay optics of adequately oversized, and we conjugate the Lyot stop with the plane of DM1, where the apodizer is located. For each ACAD configuration we test a suite of spider widths in the Lyot stop and keep Lyot stop that the open loop solution that yields the deepest open loop $10 \%$ bandwidth contrast. We then proceed to adjust the mirror shapes according to the broadband Stroke Minimization algorithm. We project our DM shapes on a set of influence functions:

$$
\delta h^{i}=\lambda_{0} \sum c_{k} f_{k}(x, y)
$$

and apply an iterative algorithm (20). At each iteration we solve the following problem:

$$
\min \sum w_{k}\left|c_{k}\right|^{2}+\left.\Gamma\left|\int_{\Delta \lambda} \int_{D H} g(\xi)\right| \mathcal{C}\left[e^{i \frac{4 \pi}{\lambda}\left(h_{0}^{2}+\delta h^{2}\right)} \mathcal{F}_{z}\left(e^{i \frac{4 \pi}{\lambda}\left(h_{0}^{1}+\delta h^{1}\right)}\right)\right] d \lambda d \xi\right|^{2}-10^{-C} \mid
$$

Note that for this study we choose a version of the Stroke Minimization algorithm that applies the same weight to each actuator $w_{k}=1$, and to each pixel $g(\xi)=1$ and seeks to improve the contrast by $10 \%$ at each iterations. Some of the limitations we find in our study are clearly driven by this conservative choice of parameters, which we will loosen in the future.

\section{FINDING OF NUMERICAL EXPERIMENT, CONCLUSION}

Figures 8, 9 and 10 presented the outcome of this numerical experiment. In the case of HST like support structure, gentle geometric remapping, the spider width in the Lyot stop is twice larger than in the initial pupil in order to minimize the contrast after diffractive propagation of the open loop ACAD commands. As a result the stroke minimization algorithm yields DM adjustments that are $\sim 80 \mathrm{~nm}$ peak to valley, and the linear loop diverges 


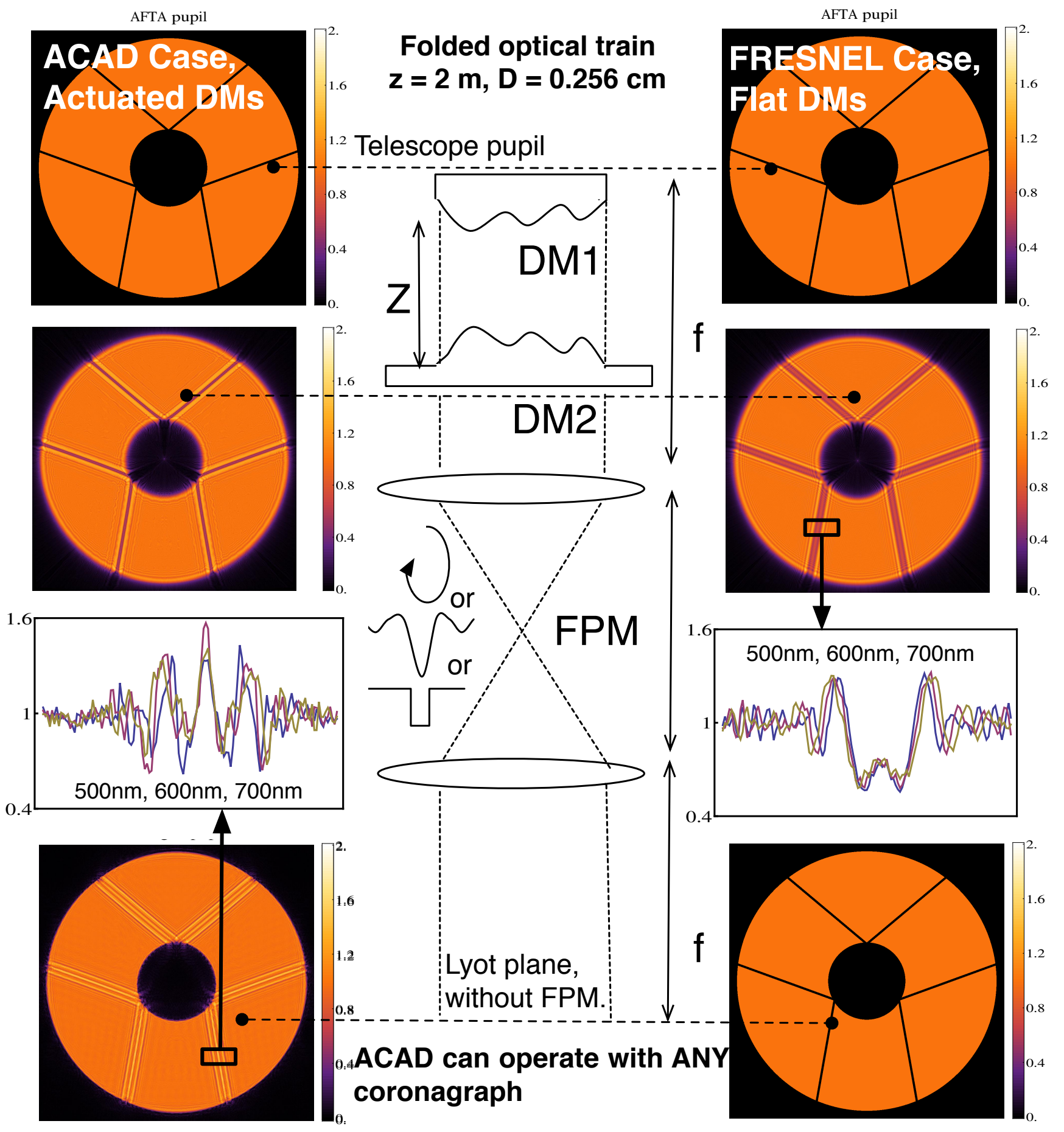

Figure 7. Simulation of ACAD in the Fresnel approximation: We simulated the propagation between DM1 and DM2 in the Fresnel regime, and simulated the rest of the coronagraphic train using the Fresnel approximation. Fresnel propagation with flat DMs simply replicates the input pupil's (top panels) deep and sharp discontinuities in the final reimaged pupil (right bottom panel). ACAD Fresnel instead transforms them into shallow and smooth mid-spatial frequency ripples (left bottom panel). The chromatic nature of these oscillations sets the ultimate bandwidth of ACAD, but unlike in PIAA coronagraphs the beam reshaping here does not amplify these oscillations any beyond normal edge ringing for out of pupil optics in the Fresnel regime (discussed in (19)). The plotted slices across the secondary support structures (middle panels) show that the amplitude ripples in the Fresnel approximation are neither bigger nor more chromatic than hard-edge induced ripples in the Fresnel approximation with flat DMs. These amplitude ripples are similar to those that can be calculated in the SR-Fresnel regime and both methods differ on the phase of the filed at DM2. Here we intentionally omit the coronagraphic mask and Lyot stop to highlight that ACAD can operate with any coronagraph. 

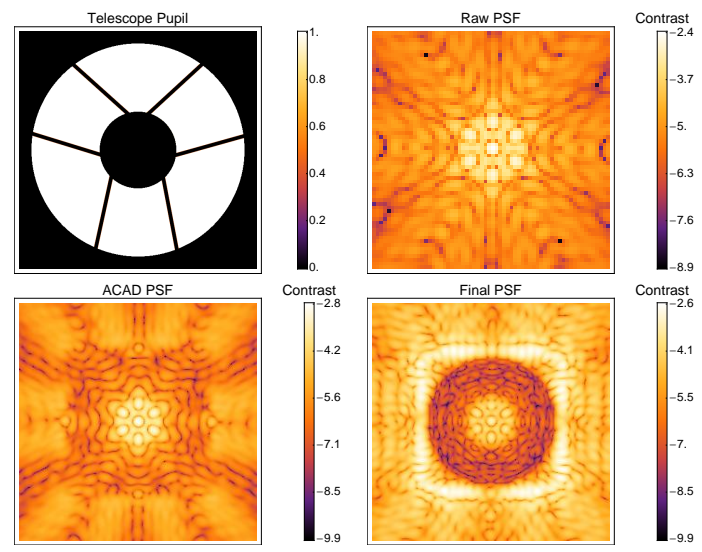

Contrast
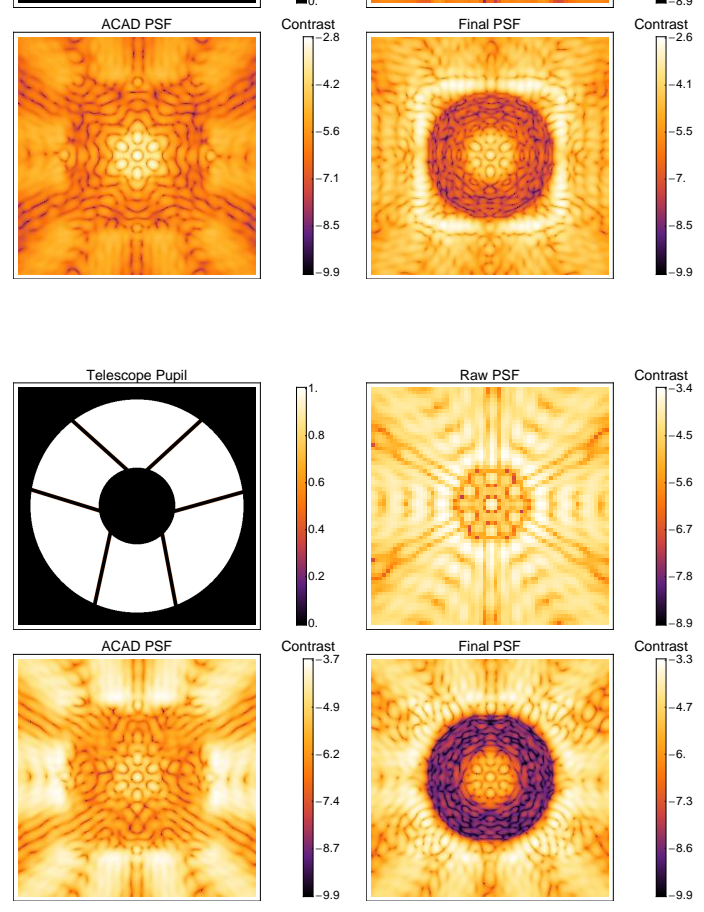
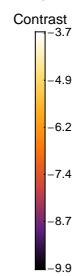

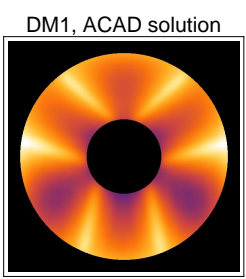

DM1, adjustment
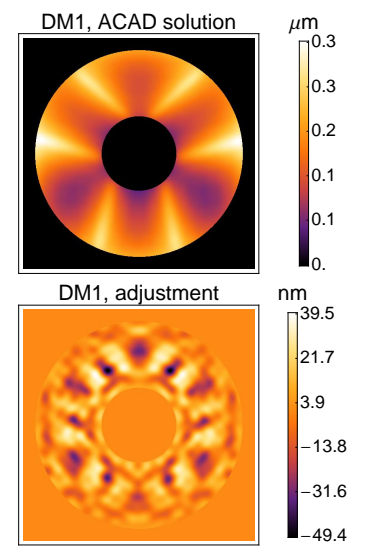

$\mathrm{nm}$
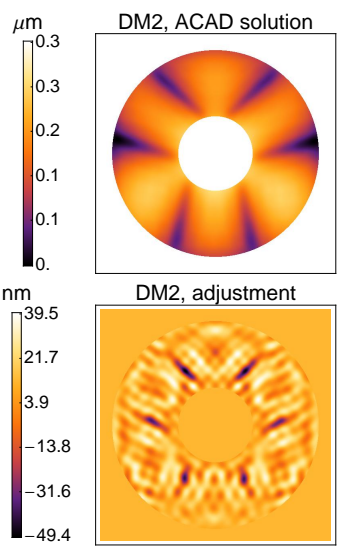

DM2, adjustment
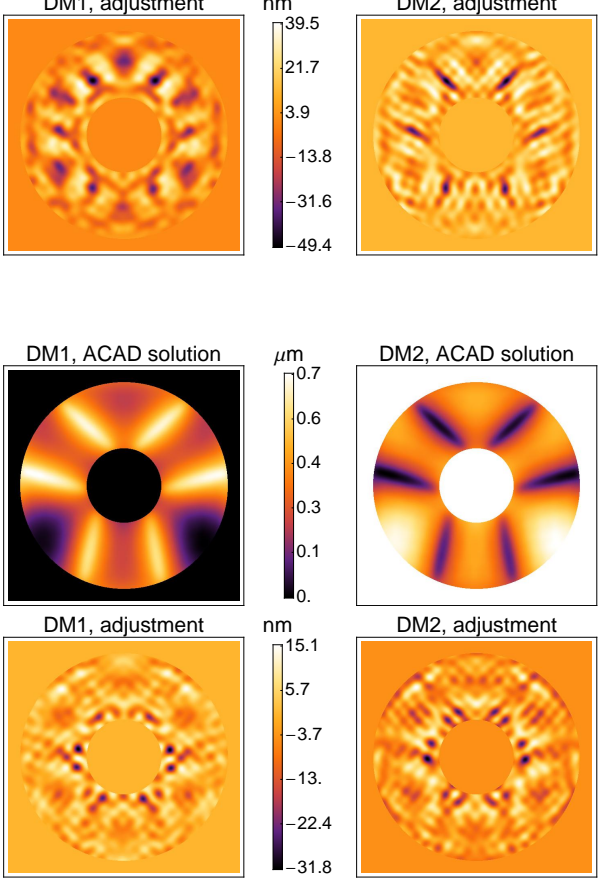

DM1, adjustment

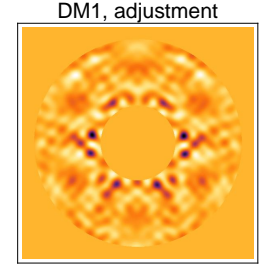

DM2, adjustment

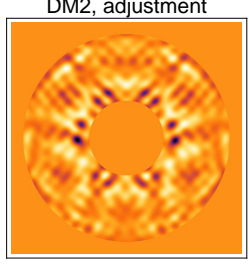

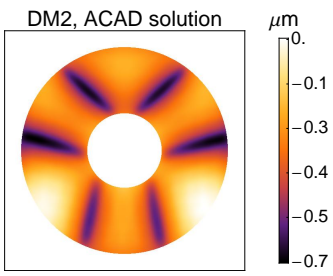
$\mathrm{nm}$

$\pi^{16.4}$

8.7

Figure 8. Top, HST like support structure, gentle geometric remapping: in this case the spider width in the Lyot stop is twice larger than in the initial pupil in order to minimize the contrast after diffractive propagation of the open loop ACAD commands. As a result the Stroke Minimization algorithm yields DM adjustments that are $\sim 80 \mathrm{~nm}$ peak to valley, and the linear loop diverges before at contrast $\sim 5 \times 10^{-8}$. Bottom, HST like support structure, aggressive geometric remapping: in this case the spider width in the Lyot stop is much smaller than in the initial pupil since the open loop ACAD solution mitigates their effect even in the presence of diffraction. This results in smaller DM adjustments and the final contrast improves to $\sim 5 \times 10^{-9}$. However, in this latter configuration, the open loop DM strokes are twice larger than in the top panel.

before at contrast $\sim 5 \times 10^{-8}$. With the same geometry and an aggressive geometric remapping the spider width in the Lyot stop is much smaller than in the initial pupil since the open loop ACAD solution mitigates their effect even in the presence of diffraction. This results in smaller DM adjustments and the final contrast improves to $\sim 5 \times 10^{-9}$. However in this latter configuration the open loop DM strokes are twice larger. For an AFTA like support structure, even with aggressive geometrical remapping, the stroke minimization algorithm yields DM adjustments that are $\sim 80 \mathrm{~nm}$ peak to valley, and the linear loop diverges very early at contrast $\sim 2 \times 10^{-7}$. In order to reach deeper contrast the actuator and pixel weights our to be adjusted in the Stroke Minimization algorithm. When the support structures are twice smaller than for HST the coronagraph contrast of $\sim 1 \times 10^{-9}$ is reached. Finally for secondary support structures of the scale of HST, TMT and E-ELT, our numerical experiment establishes that contrasts of at least $\sim 10^{-9}$ over a $10 \%$ bandwidth can be reached. However for large secondary support structures, such as for AFTA, our approach ought to be adjusted. The fact 

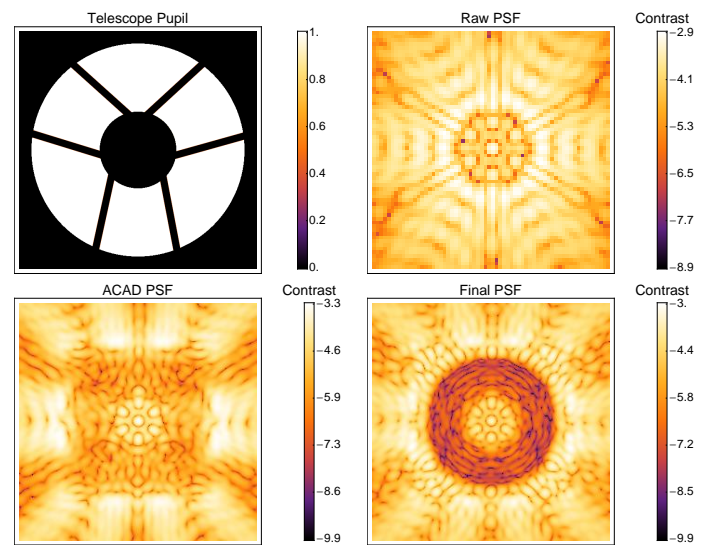

Contrast
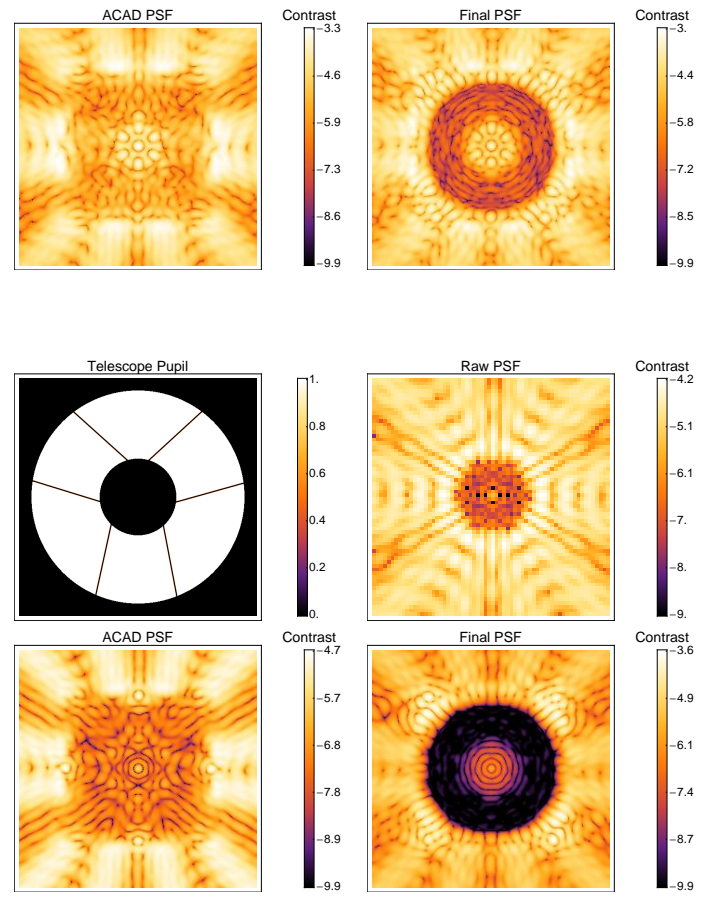

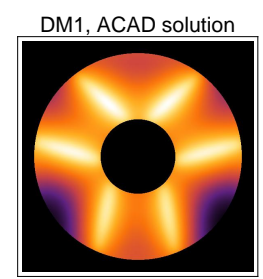

DM1, adjustment
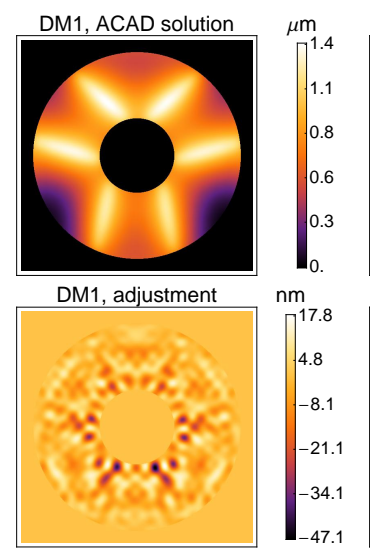

$\mathrm{nm}$
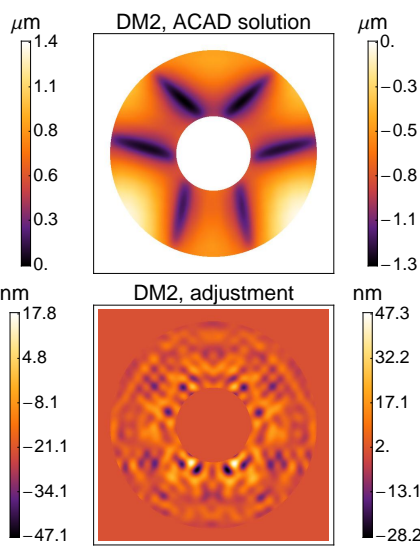

DM2, adjustment
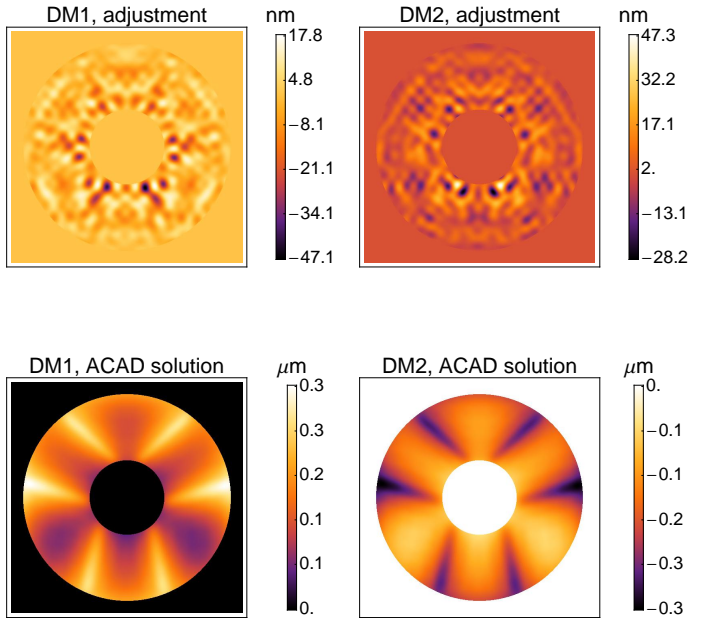

DM1, adjustment

$\mathrm{nm}^{8.3}$

DM2, adjustment

$\mathrm{nm}^{10}$.
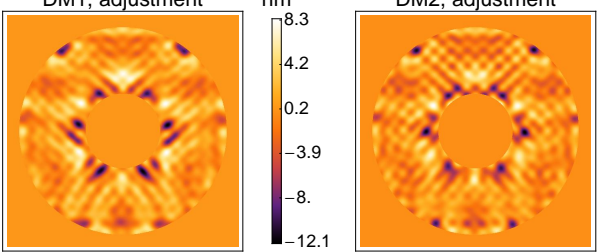

5.4

0.7

0.7

$-3.9$

-8.6
-13.2

Figure 9. Top, AFTA like support structure: even with aggressive geometrical remapping, the Stroke Minimization algorithm yields DM adjustments that are $\sim 80 \mathrm{~nm}$ peak to valley, and the linear loop diverges very early, for contrasts $\sim 2 \times 10^{-7}$. In order to reach deeper contrast the actuator and pixel weights ought to be adjusted in the Stroke Minimization algorithm. Support structure twice smaller than HST: the contrast for which the coronagraph was designed, $\sim 1 \times 10^{-9}$, is reached.

that the slope of the final contrast as a function of spider width is steeper than in the raw data suggests that more sophisticated variant of the Stroke Minimization algorithm ought to be explored in order to obtain deeper contrasts. We will present results of a more complete set of simulations, including adjustments to the algorithm when the secondary support structures are thick and bandwidths larger than $10 \%$.

\section{ACKNOWLEDGMENTS}

This material is partially based upon work supported by the National Aeronautics and Space Administration under Grant NNX12AG05G issued through the Astrophysics Research and Analysis (APRA) program . This work was performed in part under contract with the California Institute of Technology funded by NASA through the Sagan Fellowship Program executed by the NASA Exoplanet Science Institute. This material is also partially based upon work carried out under subcontract 1496556 with the Jet Propulsion Laboratory funded by NASA and administered by the California Institute of Technology. 


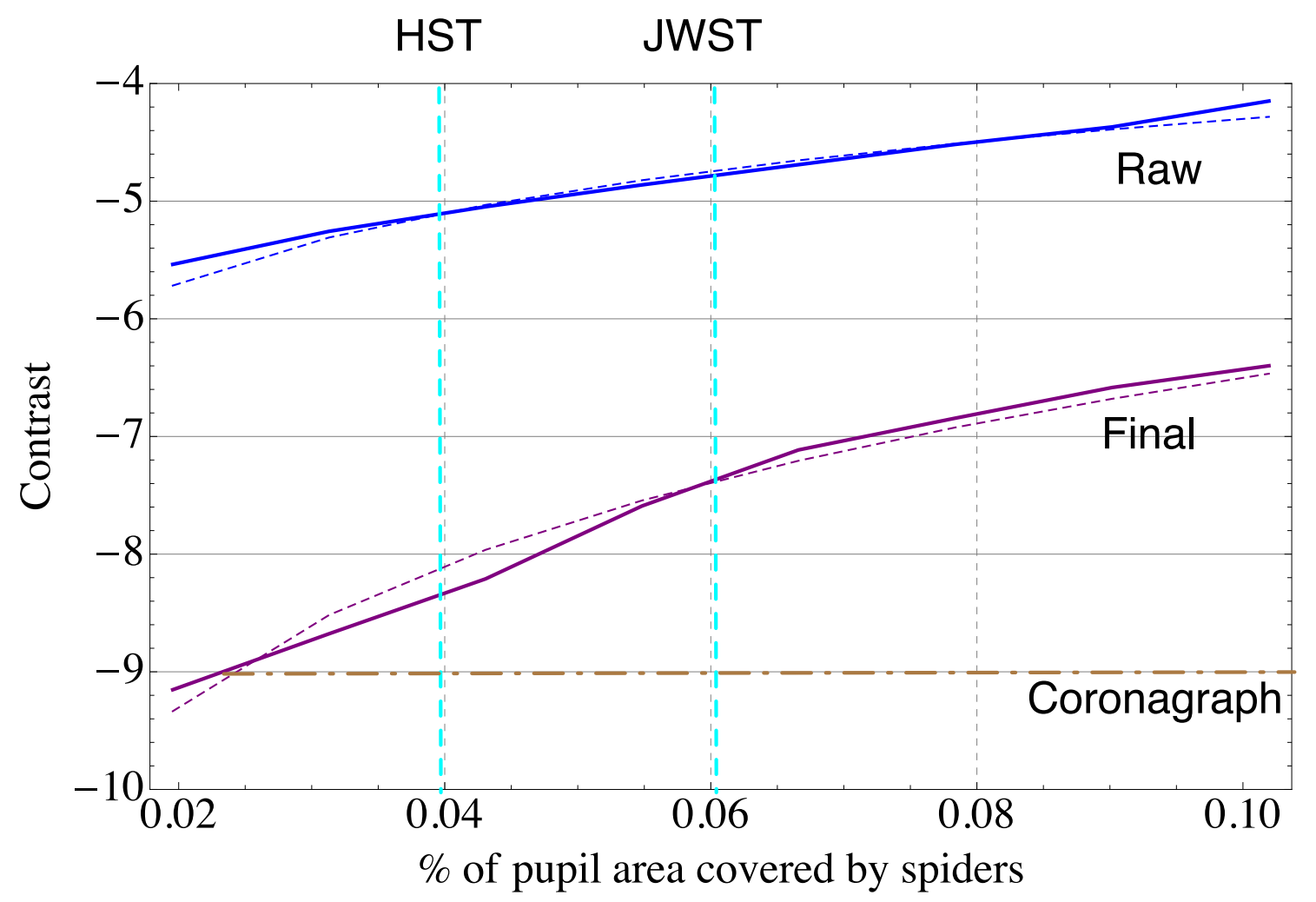

Figure 10. Final contrast as a function of the width of secondary support structures: For secondary support structures of the scale of HST, TMT and E-ELT, our numerical experiment establishes that contrasts of a few parts in $\sim 10^{-9}$ over a $10 \%$ bandwidth can be reached. However for large secondary support structures, such as AFTA, the approach in our numerical experiment ought to be adjusted. The fact that the slope of the final contrast as a function of spider width is steeper than in the raw data suggests that more sophisticated variant of the Stroke Minimization algorithm ought to be explored in order to obtain deeper contrasts.

\section{References}

[1] R. Soummer, A. Sivaramakrishnan, L. Pueyo, B. Macintosh, and B. R. Oppenheimer, "Apodized Pupil Lyot Coronagraphs for Arbitrary Apertures. III. Quasi-achromatic Solutions," ApJ 729, p. 144, Mar. 2011.

[2] B. Macintosh, M. Troy, R. Doyon, J. Graham, K. Baker, B. Bauman, C. Marois, D. Palmer, D. Phillion, L. Poyneer, I. Crossfield, P. Dumont, B. M. Levine, M. Shao, G. Serabyn, C. Shelton, G. Vasisht, J. K. Wallace, J.-F. Lavigne, P. Valee, N. Rowlands, K. Tam, and D. Hackett, "Extreme adaptive optics for the Thirty Meter Telescope," in Society of Photo-Optical Instrumentation Engineers (SPIE) Conference Series, Society of Photo-Optical Instrumentation Engineers (SPIE) Conference Series 6272, July 2006.

[3] O. Guyon, E. A. Pluzhnik, R. Galicher, F. Martinache, S. T. Ridgway, and R. A. Woodruff, "Exoplanet Imaging with a Phase-induced Amplitude Apodization Coronagraph. I. Principle," ApJ 622, pp. 744-758, Mar. 2005.

[4] R. J. Vanderbei, D. N. Spergel, and N. J. Kasdin, "Circularly Symmetric Apodization via Star-shaped Masks," ApJ 599, pp. 686-694, Dec. 2003.

[5] R. J. Vanderbei, D. N. Spergel, and N. J. Kasdin, "Spiderweb Masks for High-Contrast Imaging," ApJ 590, pp. 593-603, June 2003.

[6] N. J. Kasdin, R. J. Vanderbei, M. G. Littman, and D. N. Spergel, "Optimal one-dimensional apodizations and shaped pupils for planet finding coronagraphy," AO 44, pp. 1117-1128, Mar. 2005. 
[7] D. Mawet, L. Pueyo, D. Moody, J. Krist, and E. Serabyn, "The vector vortex coronagraph: sensitivity to central obscuration, low-order aberrations, chromaticism, and polarization," Modern Technologies in Spaceand Ground-based Telescopes and Instrumentation 7739(1), p. 773914, SPIE, 2010.

[8] M. J. Kuchner and W. A. Traub, "A Coronagraph with a Band-limited Mask for Finding Terrestrial Planets," ApJ 570, pp. 900-908, May 2002.

[9] R. Soummer, C. Aime, and P. E. Falloon, "Stellar coronagraphy with prolate apodized circular apertures," $A$ and $A$ 397, pp. 1161-1172, Jan. 2003.

[10] R. A. Brown and C. J. Burrows, "On the feasibility of detecting extrasolar planets by reflected starlight using the hubble space telescope," Icarus 87, pp. 484-497, 1990.

[11] P. J. Bordé and W. A. Traub, "High-Contrast Imaging from Space: Speckle Nulling in a Low-Aberration Regime," ApJ 638, pp. 488-498, Feb. 2006.

[12] A. Give'on, R. Belikov, S. Shaklan, and J. Kasdin, "Closed loop, dm diversity-based, wavefront correction algorithm for highcontrast imaging systems," Opt. Express 15(19), pp. 12338-12343, 2007.

[13] J. T. Trauger and W. A. Traub, "A laboratory demonstration of the capability to image an Earth-like extrasolar planet," Nature 446, pp. 771-773, Apr. 2007.

[14] O. Guyon, E. Pluzhnik, F. Martinache, J. Totems, S. Tanaka, T. Matsuo, C. Blain, and R. Belikov, "HighContrast Imaging and Wavefront Control with a PIAA Coronagraph: Laboratory System Validation," PASP 122, pp. 71-84, Jan. 2010.

[15] R. Belikov, E. Pluzhnik, F. C. Witteborn, T. P. Greene, D. H. Lynch, P. T. Zell, and O. Guyon, "Laboratory demonstration of high-contrast imaging at inner working angles $2 \lambda / \mathrm{D}$ and better," in Society of PhotoOptical Instrumentation Engineers (SPIE) Conference Series, Society of Photo-Optical Instrumentation Engineers (SPIE) Conference Series 8151, Sept. 2011.

[16] J. K. Wallace, R. Burruss, L. Pueyo, R. Soummer, C. Shelton, R. Bartos, F. Fregoso, B. Nemati, P. Best, and J. Angione, "The gemini planet imager calibration testbed," in Proceedings of the SPIE conference 7440, S. B. Shaklan, ed., Techniques and Instrumentation for Detection of Exoplanets IV 7440, SPIE, 2009.

[17] L. Pueyo, K. Wallace, M. Troy, R. Burruss, B. Macintosh, and R. Soummer, "Advanced static speckle calibration for exoplanet imaging," Adaptive Optics Systems II 7736(1), p. 77362A, SPIE, 2010.

[18] S. B. Shaklan and J. J. Green, "Reflectivity and optical surface height requirements in a broadband coronagraph. 1.Contrast floor due to controllable spatial frequencies," AO 45, pp. 5143-5153, July 2006.

[19] L. Pueyo and N. J. Kasdin, "Polychromatic Compensation of Propagated Aberrations for High-Contrast Imaging," ApJ 666, pp. 609-625, Sept. 2007.

[20] L. Pueyo, J. Kay, N. J. Kasdin, T. Groff, M. McElwain, A. Give'on, and R. Belikov, "Optimal dark hole generation via two deformable mirrors with stroke minimization," Appl. Opt. 48(32), pp. 6296-6312, 2009.

[21] T. D. Groff, A. Carlotti, and N. J. Kasdin, "Progress on broadband control and deformable mirror tolerances in a 2-DM system," in Society of Photo-Optical Instrumentation Engineers (SPIE) Conference Series, Society of Photo-Optical Instrumentation Engineers (SPIE) Conference Series 8151, Sept. 2011.

[22] S. B. Shaklan, J. J. Green, and D. M. Palacios, "The terrestrial planet finder coronagraph optical surface requirements," in Space Telescopes and Instrumentation I: Optical, Infrared, and Millimeter. Edited by Mather, John C.; MacEwen, Howard A.; de Graauw, Mattheus W. M.. Proceedings of the SPIE, Volume 6265, pp. 62651I (2006)., Presented at the Society of Photo-Optical Instrumentation Engineers (SPIE) Conference 6265, July 2006. 
[23] J. E. Krist, R. Belikov, L. Pueyo, D. P. Mawet, D. Moody, J. T. Trauger, and S. B. Shaklan, "Assessing the performance limits of internal coronagraphs through end-to-end modeling: a NASA TDEM study," in Society of Photo-Optical Instrumentation Engineers (SPIE) Conference Series, Society of Photo-Optical Instrumentation Engineers (SPIE) Conference Series 8151, Sept. 2011.

[24] L. Pueyo and C. Norman, "High-contrast Imaging with an Arbitrary Aperture: Active Compensation of Aperture Discontinuities," 769, p. 102, June 2013.

[25] R. Soummer, "Apodized Pupil Lyot Coronagraphs for Arbitrary Telescope Apertures," ApJl 618, pp. L161L164, Jan. 2005.

[26] D. P. e. a. Mawet, "Ring-apodized vortex coronagraphs for obscured telescopes. i. transmissive ring apodizers.," ApJS Accpeted, 2013.

[27] R. J. Vanderbei and W. A. Traub, "Pupil Mapping in Two Dimensions for High-Contrast Imaging," ApJ 626, pp. 1079-1090, June 2005.

[28] O. Guyon, "Phase-induced amplitude apodization of telescope pupils for extrasolar terrestrial planet imaging," A and A 404, pp. 379-387, June 2003.

[29] W. A. Traub and R. J. Vanderbei, "Two-Mirror Apodization for High-Contrast Imaging," ApJ 599, pp. 695701, Dec. 2003.

[30] L. Pueyo, N. J. Kasdin, A. Carlotti, and R. Vanderbei, "Design of Phase Induced Amplitude Apodization Coronagraphs Over Square Apertures," ApJs 195, p. 25, Aug. 2011.

[31] P. Martinez, A. Boccaletti, M. Kasper, C. Cavarroc, N. Yaitskova, T. Fusco, and C. Vérinaud, "Comparison of coronagraphs for high-contrast imaging in the context of extremely large telescopes," $A$ and $A \mathbf{4 9 2}$, pp. 289-300, Dec. 2008.

[32] R. J. Vanderbei, "Diffraction Analysis of Two-dimensional Pupil Mapping for High-Contrast Imaging," ApJ 636, pp. 528-543, Jan. 2006.

[33] E. Pluzhnik, O. Guyon, S. Ridgway, R. Martinache, R. Woodruff, C. Blain, and R. Galicher, "Exoplanets imaging with a phase-induced amplitude apodization coronagraph-iii. hybrid approach: Optical design and diffraction analysis," Submitted to The Astrophysical Journal, 2006. (astro-ph/0512421).

[34] J. E. Krist, L. Pueyo, and S. B. Shaklan, "Practical numerical propagation of arbitrary wavefronts through piaa optics," Space Telescopes and Instrumentation 2010: Optical, Infrared, and Millimeter Wave 7731(1), p. $77314 \mathrm{~N}$, SPIE, 2010. 